\title{
Carbono da biomassa microbiana em solo cultivado com soja sob diferentes sistemas de manejo nos Cerrados
}

\author{
Kátia Sueli Sivek Perez ${ }^{(1)}$, Maria Lucrécia Gerosa Ramos ${ }^{(2)}$ e Concepta McManus ${ }^{(2)}$
}

(1)Fundação Mokiti Okada, Caixa Postal 033, CEP 13537-000 Ipeúna, SP. (2)Universidade de Brasília, Fac. de Agronomia e Medicina Veterinária, Caixa Postal 04508, CEP 70910-970 Brasília, DF. E-mail lucrecia@unb.br, concepta@unb.br

Resumo - O objetivo deste trabalho foi quantificar o carbono da biomassa microbina de solo, cultivado com soja em diferentes sistemas de manejo. Os sistemas de manejo foram: semeadura direta, uma gradagem, subsolagem e duas gradagens, realizadas num Latossolo Vermelho-Amarelo argiloso. As amostras de solo foram coletadas em cinco profundidades $(0-5,5-10,10-20,20-30$ e 30-40 cm) e em quatro épocas (antes do preparo do solo, 30 dias após a germinação, floração e após a colheita da soja). Foram coletadas, também, amostras de solo na mesma profundidade e na mesma época, em uma área de vegetação nativa (Cerrado sensu strictu), adjacente ao experimento. A subsolagem apresentou os maiores valores de carbono aos 30 dias após a germinação $\left(865,7 \mathrm{mg} \mathrm{kg}^{-1} \mathrm{de}^{-}\right.$ solo). Este valor foi reduzido para $80,3 \mathrm{mg} \mathrm{kg}^{-1}$ de solo na floração. Os valores de carbono na semeadura direta mantiveram-se mais estáveis, principalmente na camada de 0-20 cm. As camadas de 0-5 e 5-10 cm apresentaram diferença na maioria das épocas estudadas e das demais camadas. A subsolagem mostrou o menor valor do carbono orgânico do solo, após a colheita da soja. Não houve correlação entre a relação carbono da biomassa microbiana/carbono orgânico e os nutrientes do solo na subsolagem.

Termos para indexação: Glycine max, carbono orgânico, indicador de qualidade de solo.

\section{Microbial biomass carbon in soil cultivated with soybean, under different management systems in Cerrado}

Abstract - The aim of this study was to quantify soil microbial biomass carbon in a soybean crop under different soil management systems: no-tillage, single harrowing, subsoiling and two harrowing, on a clay Red-Yellow Latossol in the Cerrado region. Soils were studied at five depths: 0-5, 5-10, 10-20, 20-30 and 30-40 cm. Four periods were observed: before soil preparation; 30 days after germination; flowering stage and after harvesting soybean plants. The same measurements were taken under the same conditions in an area of native cerrado vegetation, adjacent to the experiment (Cerrado sensu strictu). Subsoiling showed highest carbon values 30 days after germination ( $865.7 \mathrm{mg} \mathrm{kg}^{-1}$ of soil). This was reduced to less than $10 \%\left(80.3 \mathrm{mg} \mathrm{kg}^{-1}\right)$ at flowering. The carbon values were more stable in the no-tillage system, mainly in the $0-20 \mathrm{~cm}$ layer. The layers $0-5 \mathrm{~cm}$ and $5-10 \mathrm{~cm}$ showed significantly higher carbon values compared with other layers in most of the studied periods and depths. Subsoiling showed the lowest soil organic carbon level after soybean harvesting. Only in the system with subsoiling there was no significant correlation between the microbial carbon:organic carbon index and soil nutrients.

Index terms: Glycine max, organic carbon, soil quality indicator.

\section{Introdução}

O solo é um sistema aberto e concentra resíduos orgânicos de origem vegetal, animal e os produtos das transformações destes resíduos; a vegetação é a principal responsável pela deposição de materiais orgânicos no solo. O tipo de vegetação e as condições ambientais são fatores que determinam a quantidade e a qualidade do material que se deposita no solo, influenciando a heterogeneidade e a taxa de decomposição do material depositado a superfície (Moreira \& Siqueira, 2002). A decomposição destes materiais depende dos processos de transformação da matéria orgânica pelos microrganismos do solo, por meio dos quais pode-se mensurar a qualidade do solo, determinando-se os valores do carbono da biomassa microbiana (Sparling, 1992). 
Na região do Cerrado, grande parte da vegetação natural está sendo substituída por culturas anuais e pas tagens. O preparo intensivo da terra, nessa região, implica o revolvimento do solo repetidas vezes antes da implantação de cada cultura, ocasionando intensa perturbação do solo, aumentando a mineralização da matéria orgânica e promovendo a erosão e o aquecimento global pela emissão do dióxido de carbono (Urquiaga et al., 1999).

A matéria orgânica do solo representa o principal reservatório de energia para os microrganismos e de nutrientes para as plantas. O declínio ou acréscimo da matéria orgânica do solo serve para mensurar a preservação dos ecossistemas naturais e os desequilíbrios dos agroecossistemas; ou seja, é utilizado como critério na avaliação da sua sustentabilidade (Kaiser et al., 1995).

O plantio direto, em relação ao plantio convencional, permite elevar os teores de matéria orgânica no solo. No plantio direto e no cultivo mínimo, a semeadura é realizada sob resíduos da cultura anterior sem movimentação do solo, exceto na linha de semeadura, ocorrendo acúmulo de resíduos vegetais. Porém, no plantio convencional são utilizadas operações como aração e gradagem e, geralmente, o solo fica descoberto (Wardle, 1994).

A biomassa microbiana é a fração viva da matéria orgânica do solo composta por bactérias, fungos, actinomicetos, protozoários e algas. Ela é um importante componente na avaliação da qualidade do solo porque atua nos processos de decomposição natural interagindo na dinâmica dos nutrientes e regeneração da estabilidade dos agregados (Franzluebbers et al., 1999). A biomassa microbiana é influenciada pelas variações sazonais de umidade e temperatura, pelo manejo do solo, pelo cultivo e, também, pelos resíduos vegetais. Representa pequena parte da fração ativa da matéria orgânica (De Luca, 1998), constituindo apenas 2\% a 5\% do C orgânico do solo. Apesar disso, é mais sensível que os teores de $\mathrm{C}$ orgânico e $\mathrm{N}$ total para aferir alterações na matéria orgânica causadas pelas práticas de cultivo (Gama-Rodrigues, 1999).

A biomassa microbiana pode ser utilizada como um indicador biológico ou como índice de adequação de sustentabilidade de sistemas de produção (Anderson \& Domsch, 1993) e, geralmente, apresenta forte correlação com a matéria orgânica do solo, ou seja, reflete mudanças na concentração de matéria orgânica. A razão carbono microbiano e carbono orgânico indica a qualidade da matéria orgânica (Wardle, 1994). De acordo com Sparling (1992), pode-se monitorar a dinâmica da matéria orgânica do solo usando-se a razão carbono microbiano $\left(\mathrm{C}_{\mathrm{mic}}\right)$ :carbono orgânico $\left(\mathrm{C}_{\mathrm{org}}\right)$

O objetivo do presente trabalho foi quantificar o carbono da biomassa microbiana do solo, cultivado com soja em diferentes sistemas de manejo.

\section{Material e Métodos}

O experimento foi realizado numa área do Distrito Federal, cultivada com soja por 20 anos, sob plantio convencional, em um Latossolo Vermelho-Amarelo argiloso. No ano agrícola 2000/2001, após a colheita da soja (Glycine max), foi semeado o milheto (Pennisetum glaucum L.) em toda a área, que serviu como cobertura vegetal. Na safra 2001/2002, durante o estabelecimento do experimento aplicou-se 1,0 t ha-1 de calcário dolomítico e $320 \mathrm{~kg} \mathrm{ha}^{-1}$ de fosfato natural de Arade; $50 \mathrm{~kg} \mathrm{ha}^{-1}$ de sulfato de potássio e $30 \mathrm{~kg} \mathrm{ha}^{-1}$ de nutriBokashi (N, 3\%; P, 2\%; K, 1,4\%; Ca, 2,2\%; Mg, 1,1\%; $\mathrm{Mn}, 0,018 \%$; Zn, 0,011\%; Fe, 0,090\%; B, 0,020\%; Cu, $0,010 \%$ e $\mathrm{pH}, 6,0)$. As sementes de soja (cultivar Vitória) foram submetidas à inoculação com $600 \mathrm{~g}$ de inoculante $/ 50 \mathrm{~kg}$ de sementes, com uma população de $10^{9} \mathrm{UFC} / \mathrm{g}$ de inoculante.

Neste período, a área foi dividida em três partes de 0,5 ha $(50 \times 100 \mathrm{~m})$ e foram realizados os seguintes preparos de solo: semeadura direta da soja (SD); uma subsolagem e duas gradagens (SG) e uma gradagem (GR).

Foram coletadas amostras de solo nos tratamentos e em uma vegetação nativa (Cerrado sensu strictu), adjacente ao experimento. Na mata, foi delimitada uma área de 50x100 m, onde foram feitas as coletas. As amostras de solo foram coletadas nas profundidades de 0-5, 5-10, 10-20, 20-30 e 30-40 cm e em quatro épocas do ciclo da cultura da soja, ou seja, antes do preparo do solo, 30 dias após a germinação, na floração, e após a colheita da cultura. Em cada área foi projetada uma linha imaginária em diagonal e as coletas foram feitas em três pontos eqüidistantes, representando as repetições. Em cada ponto foram coletadas cinco subamostras obtendo-se uma amostra composta de cada profundidade. Logo após a coleta, as amostras foram mantidas em caixas de isopor e resfriadas durante o transporte até o laboratório, onde foram preservadas em câmara fria a $4^{\circ} \mathrm{C}$.

Como as avaliações são repetidas no espaço e no tempo, utilizou-se a análise proposta por Milliken \& Johnson (1993) e Hinkelman \& Kempthorne (1994), em 
que as profundidades podem ser consideradas como subparcelas e as épocas do ano como subsubparcelas. Os valores do carbono da biomassa microbiana do solo foram estimados pelo método de fumigação e incubação (Jenkinson \& Powlson, 1976). O teor de carbono orgânico do solo foi avaliado pelo método da oxidação por via úmida (Walkley \& Black, 1934). O carbono da biomassa microbiana foi calculado pela fórmula $\mathrm{B}=(\mathrm{F}-\mathrm{NF}) \mathrm{k}_{\mathrm{c}}{ }^{-1}$, em que $\mathrm{F}$ e NF representam o carbono total do $\mathrm{CO}_{2}$ que foi liberado das amostras fumigadas e não fumigadas, respectivamente; $\mathrm{k}_{\mathrm{c}}$ é uma constante $(0,41)$, representando a proporção do carbono da biomassa microbiana morta que é convertido em $\mathrm{CO}_{2}$ durante o período de incubação.

Os dados foram analisados pela PROC MIXED GLM do programa estatístico SAS-Statistic Analitical System (SAS Institute, 1997). As fontes de variação foram os tratamentos (parcela), profundidades (subparcelas), épocas de amostragem (subsubparcelas) e suas interações. Os efeitos principais foram separados pelo teste de Tukey a $5 \%$ de probabilidade.

\section{Resultados e Discussão}

A análise de regressão mostrou efeito linear do carbono orgânico do solo nas profundidades estudadas, sob solo de Cerrado e nos diferentes manejos de solo, durante o ciclo da soja (Figura 1).

$\mathrm{Na}$ primeira coleta, antes do preparo de solo, os resultados revelaram teores de carbono orgânico $\left(\mathrm{C}_{\mathrm{org}}\right)$ semelhantes, variando de 12,1 a $12,6 \mathrm{~g} \mathrm{~kg}^{-1}$ de solo, não havendo diferença significativa nos diferentes manejos de solo e no Cerrado. Este último apresentou teores superiores em todas as épocas amostradas (Tabela 1).

Aos 30 dias após a germinação (DAG), o solo sob cerrado apresentou maior teor de $\mathrm{C}_{\text {org }}$ em relação aos manejos de solo GR (gradagem), SG (subsolagem) e SD (semeadura direta). Não houve diferença significativa entre os sistemas de preparo.

$\mathrm{Na}$ floração, os teores de $\mathrm{C}_{\text {org }}$ no cerrado mantiveram-se superiores aos sistemas de preparo de solo. Nestes, o preparo de solo com SG apresentou teores de carbono maiores que com GR e SD. Após a colheita da soja, o solo que foi subsolado apresentou teor de $\mathrm{C}_{\text {org }}$
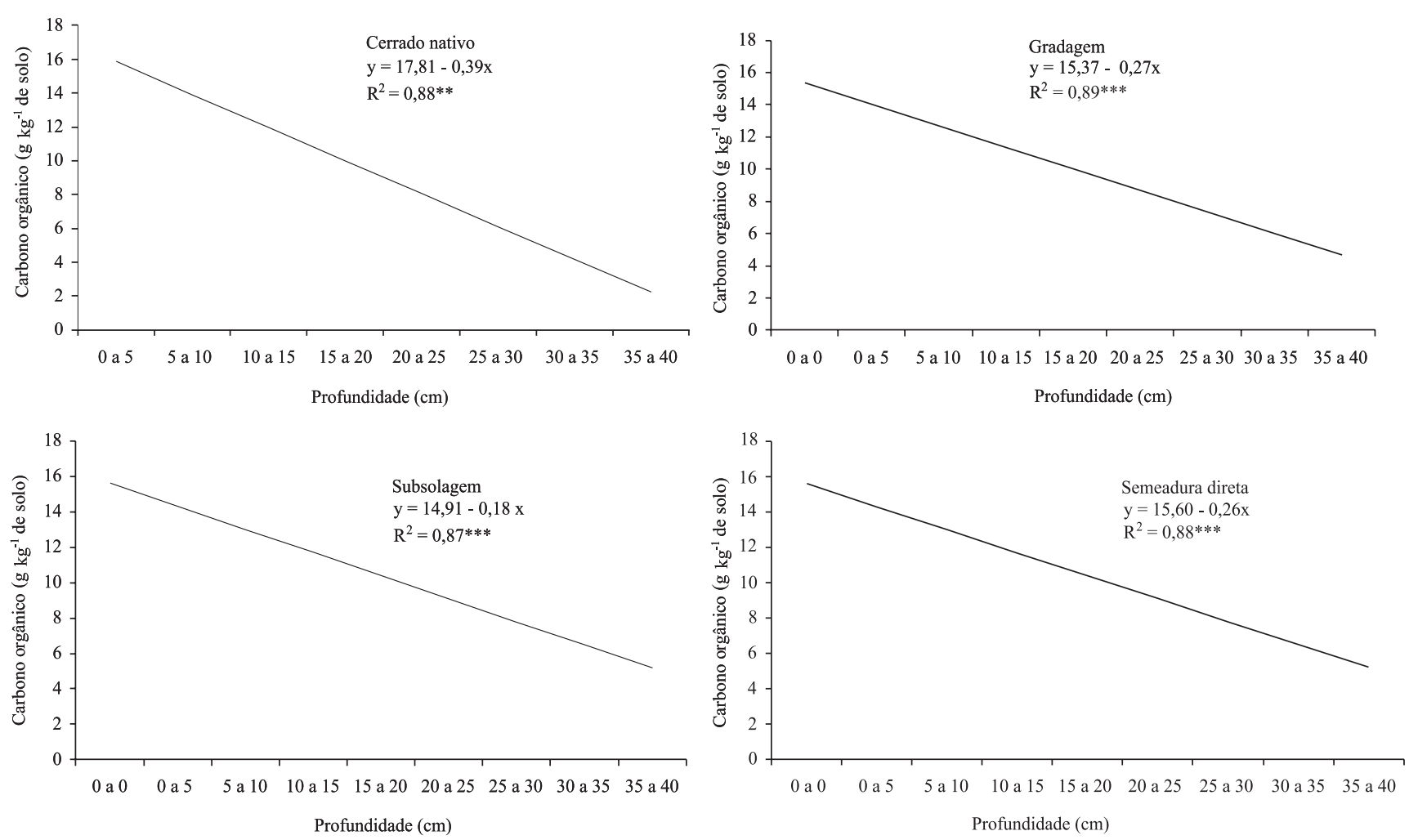

Figura 1. Relação entre o carbono orgânico e a profundidade do solo no cerrado nativo, no solo sob gradabem, no solo sob subsolagem e no solo sob semeadura direta. 
menor que o dos tratamentos que receberam gradagem, semeadura direta e o solo sob cerrado.

A distribuição do $\mathrm{C}_{\text {org }}$ foi decrescente no solo de cerrado e com diferentes manejos, ao longo do perfil, mostrando que os menores teores estão associados ao processo de perturbação do solo (Figura 1). Os valores de $\mathrm{C}_{\text {org }}$ reduziram-se com a profundidade em todas as épocas estudadas; a acumulação de $\mathrm{C}_{\text {org }}$ foi maior na camada superficial decrescendo com a profundidade (Tabela 2). Em solo não perturbado, o acúmulo de carbono na camada $0-5 \mathrm{~cm}$ foi maior do que nos solos cultivados (Tabela 3). D'Andréa et al. (2002) encontraram valores semelhantes de carbono orgânico em solo de Cerrado, ou seja, 18, 12 e $8 \mathrm{~g} \mathrm{~kg}^{-1}$ de solo, nas profundidades $0-10,10-20$ e $30-40 \mathrm{~cm}$, respectivamente. Nas camadas 5-10 e 10-20 cm, não houve diferença significativa em cada sistema de preparo (Tabela 3 ) Nas profundidades $20-30$ e $30-40 \mathrm{~cm}$, o cerrado mostrou os maiores teores, enquanto que o solo cultivado apresentou variações de pequena intensidade, não havendo diferença entre o GR, SG e SD.

Os resíduos orgânicos depositados no solo, após a decomposição, são essenciais no processo de adição e perda de C orgânico do solo (Doran, 1980).

Nos solos cultivados, a adição e a perda de C orgânico são influenciadas pelo manejo do solo. Os resíduos

Tabela 1. Carbono orgânico $\left(\mathrm{C}_{\text {org }}\right)$ e da biomassa microbiana $\left(\mathrm{C}_{\text {mic }}\right)$ e relação porcentual entre essas duas variáveis, em razão dos sistemas de preparo do solo e das épocas do ciclo da cultura da $\operatorname{soja}^{(1)}$.

\begin{tabular}{|c|c|c|c|c|}
\hline $\begin{array}{l}\text { Sistema de } \\
\text { preparo }^{(2)}\end{array}$ & $\begin{array}{l}\text { Antes do } \\
\text { preparo do } \\
\text { solo }\end{array}$ & $\begin{array}{l}\text { Trinta dias } \\
\text { após a } \\
\text { germinação }\end{array}$ & Floração & $\begin{array}{l}\text { Após a } \\
\text { colheita }\end{array}$ \\
\hline & \multicolumn{4}{|c|}{ Carbono orgânico $\left(\mathrm{g} \mathrm{kg}^{-1}\right.$ de solo $)$} \\
\hline Cerrado & $12,6 \mathrm{a}$ & $13,7 \mathrm{a}$ & $12,5 \mathrm{a}$ & $12,8 \mathrm{a}$ \\
\hline GR & $12,2 \mathrm{a}$ & $12,0 \mathrm{~b}$ & & $12,3 \mathrm{a}$ \\
\hline SG & $12,1 \mathrm{a}$ & $11,9 \mathrm{~b}$ & $11,8 \mathrm{~b}$ & $11,8 \mathrm{~b}$ \\
\hline \multirow[t]{2}{*}{ SD } & $12,3 \mathrm{a}$ & $12,2 b$ & $11,7 \mathrm{bc}$ & $12,3 \mathrm{a}$ \\
\hline & \multicolumn{4}{|c|}{ Carbono da biomassa microbiana $\left(\mathrm{mg} \mathrm{kg}^{-1}\right.$ de solo) } \\
\hline Cerrado & $227,1 \mathrm{a}$ & $175,2 \mathrm{~b}$ & $201,9 \mathrm{a}$ & $187,3 \mathrm{a}$ \\
\hline GR & $115,2 \mathrm{~b}$ & $157,8 \mathrm{~b}$ & $97,0 \mathrm{~b}$ & $65,9 \mathrm{~b}$ \\
\hline SG & $91,4 \mathrm{~b}$ & $865,7 \mathrm{a}$ & $80,3 \mathrm{~b}$ & $54,2 \mathrm{~b}$ \\
\hline SD & $77,4 \mathrm{~b}$ & $201,5 b$ & $71,4 \mathrm{~b}$ & $153,0 \mathrm{a}$ \\
\hline \multicolumn{5}{|c|}{$\left(\mathrm{C}_{\mathrm{mic}}: \mathrm{C}_{\mathrm{org}}\right)$} \\
\hline Cerrado & $1,6 \mathrm{a}$ & & $1,4 \mathrm{a}$ & $1,3 a$ \\
\hline GR & $0,9 b$ & $1,3 b c$ & $0,7 \mathrm{~b}$ & $0,5 \mathrm{~b}$ \\
\hline SG & $0,6 \mathrm{~b}$ & $7,5 \mathrm{a}$ & $0,8 \mathrm{~b}$ & $0,4 \mathrm{~b}$ \\
\hline SD & $0,7 \mathrm{~b}$ & $1,6 \mathrm{~b}$ & $0,6 \mathrm{~b}$ & $1,1 \mathrm{a}$ \\
\hline
\end{tabular}

(1)Para cada variável, médias seguidas pela mesma letra, na coluna, não diferem entre si pelo teste de Tukey a $5 \%$ de probabilidade. ${ }^{(2)}$ Cerrad sensu stricu, GR. una gradagen, SG. una subsolagem e duas gradagen D: semeadura direta da soja, sem revolvimento do solo. culturais, as plantas de cobertura e os adubos verdes são incorporados e distribuídos nos perfis dos solos, de acordo com a atuação do implemento. Os implementos utilizados, subsolador e grade pesada, fragmentam os agregados do solo, expondo a matéria orgânica do solo (MOS) fisicamente protegida ao ataque dos microrganismos do solo, reduzindo os teores de $\mathrm{C}$ no solo (Tisdall \& Oades, 1982). No Rio Grande do Sul, Bayer \& Mielniczuk (1999) observaram que os métodos convencionais de preparo de solo com arações e gradagens, associados a sistemas de monocultura com baixo retorno de resíduos vegetais, resultaram em menor quantidade de carbono orgânico. A análise de regressão mostrou efeito quadrático entre o carbono da biomassa microbiana do solo $\left(\mathrm{C}_{\mathrm{mic}}\right)$ sob cerrado e as profundidades estudadas (Figura 2).

Na primeira amostragem, antes do preparo do solo, o carbono da biomassa microbiana do cerrado mostrou valor superior em relação aos três manejos do solo (Tabela 1). Isso evidencia que, em ecossistemas não perturbados, a deposição de resíduos orgânicos mantém os valores do $\left(\mathrm{C}_{\text {mic }}\right)$ e, nos ecossistemas perturbados pelas diferentes práticas agrícolas, esses valores são alterados.

Tabela 2. Carbono orgânico $\left(\mathrm{C}_{\mathrm{org}}\right)$ e da biomassa microbiana $\left(C_{\text {mic }}\right)$ e relação porcentual entre essas duas variáveis, em razão das profundidades do solo e das épocas do ciclo da cultura da $\operatorname{soja}^{(1)}$.

\begin{tabular}{ccccc}
\hline $\begin{array}{c}\text { Profundidades } \\
(\mathrm{cm})\end{array}$ & $\begin{array}{c}\text { Antes do } \\
\text { preparo } \\
\text { do solo }\end{array}$ & $\begin{array}{c}\text { Trinta dias } \\
\text { após } \\
\text { a germinação }\end{array}$ & Floração & $\begin{array}{c}\text { Após a } \\
\text { colheita }\end{array}$ \\
\hline \multicolumn{5}{c}{ Carbono orgânico $\left(\mathrm{g} \mathrm{kg}^{-1}\right.$ de solo $)$} \\
$0-5$ & $15,5 \mathrm{a}$ & $16,3 \mathrm{a}$ & $15,1 \mathrm{a}$ & $16,1 \mathrm{a}$ \\
$5-10$ & $14,2 \mathrm{~b}$ & $14,6 \mathrm{~b}$ & $13,8 \mathrm{~b}$ & $14,6 \mathrm{~b}$ \\
$10-20$ & $12,3 \mathrm{c}$ & $12,6 \mathrm{c}$ & $12,0 \mathrm{c}$ & $12,7 \mathrm{c}$ \\
$20-30$ & $10,6 \mathrm{~d}$ & $10,5 \mathrm{~d}$ & $10,1 \mathrm{~d}$ & $10,1 \mathrm{~d}$ \\
$30-40$ & $8,9 \mathrm{e}$ & $8,3 \mathrm{e}$ & $8,1 \mathrm{e}$ & $8,1 \mathrm{e}$ \\
\hline \multicolumn{5}{c}{ Carbono da biomassa microbiana $\left(\mathrm{mg} \mathrm{kg}^{-1}\right.$ de solo $)$} \\
$0-5$ & $188,8 \mathrm{a}$ & $448,3 \mathrm{a}$ & $182,1 \mathrm{a}$ & $230,6 \mathrm{a}$ \\
$5-10$ & $226,8 \mathrm{a}$ & $416,5 \mathrm{a}$ & $169,1 \mathrm{ab}$ & $190,3 \mathrm{a}$ \\
$10-20$ & $102,9 \mathrm{~b}$ & $336,5 \mathrm{~b}$ & $124,2 \mathrm{~b}$ & $83,2 \mathrm{~b}$ \\
$20-30$ & $52,7 \mathrm{~b}$ & $293,6 \mathrm{bc}$ & $57,4 \mathrm{c}$ & $49,4 \mathrm{bc}$ \\
$30-40$ & $67,6 \mathrm{~b}$ & $255,3 \mathrm{c}$ & $30,4 \mathrm{c}$ & $22,1 \mathrm{c}$ \\
\hline \multicolumn{5}{c}{$\left(\mathrm{C}_{\text {mic }}\right.$ : $\left.\mathrm{C}_{\text {org }}\right)$} \\
$0-5$ & $1,2 \mathrm{~b}$ & $2,9 \mathrm{a}$ & $1,2 \mathrm{a}$ & $1,4 \mathrm{a}$ \\
$5-10$ & $1,6 \mathrm{a}$ & $2,9 \mathrm{a}$ & $1,2 \mathrm{a}$ & $1,3 \mathrm{a}$ \\
$10-20$ & $0,8 \mathrm{bc}$ & $2,7 \mathrm{a}$ & $1,0 \mathrm{a}$ & $0,7 \mathrm{bc}$ \\
$20-30$ & $0,5 \mathrm{c}$ & $2,8 \mathrm{a}$ & $0,6 \mathrm{~b}$ & $0,5 \mathrm{bc}$ \\
$30-40$ & $0,8 \mathrm{c}$ & $3,2 \mathrm{a}$ & $0,4 \mathrm{~b}$ & $0,3 \mathrm{c}$ \\
\hline
\end{tabular}

(1)Para cada variável, médias seguidas pela mesma letra, na coluna, não diferem entre si pelo teste de Tukey a 5\% de probabilidade. 
Na segunda amostragem, aos 30 dias após a germinação, o $\left(\mathrm{C}_{\text {mic }}\right)$ na subsolagem ( $\mathrm{SG}$ ) foi 4,9 vezes maior que o do solo de cerrado, 4,3 vezes maior que o do solo $\mathrm{SD}$ e 5,5 vezes maior que o do solo com GR. No solo com $\mathrm{SG}$ houve um drástico aumento $\mathrm{C}_{\text {mic }}$, devido à homogeneização dos resíduos vegetais no perfil.

$\mathrm{Na}$ floração da cultura, $\mathrm{o}\left(\mathrm{C}_{\mathrm{mic}}\right)$ na subsolagem reduziu para $80,26 \mathrm{mg} \mathrm{kg}^{-1}$ (Tabela 1). Dessa forma, os resultados mostram o efeito da subsolagem nos atributos biológicos do solo. Após a colheita, $\mathrm{o}_{\mathrm{mic}}$ da $\mathrm{SD}$ foi o que mais se aproximou do cerrado, mostrando que a perturbação mínima do solo leva a maiores valores no $\mathrm{C}_{\text {mic }}$. Nesta época, o $\mathrm{SG}$ apresentou valor do $\mathrm{C}_{\text {mic }} 3,4$ vezes menor que no cerrado e 2,8 vezes menor que na SD.

Nas camadas $0-5$ e 5-10 cm não ocorreram diferenças significativas do $\mathrm{C}_{\text {mic }}$ nas épocas estudadas (Tabela 2). Nas épocas estudadas, exceto na floração, estas duas profundidades diferiram das camadas inferiores do perfil. Os valores decrescem com o aumento da profundidade em todas as épocas. Nas condições de mata nativa, tanto a deposição de resíduos orgânicos, quanto a grande quantidade de raízes, estimulam as atividades da microbiota do solo, principalmente nas camadas superficiais. Resultados semelhantes foram ob-

Tabela 3. Carbono orgânico $\left(\mathrm{C}_{\mathrm{org}}\right)$ e da biomassa microbiana $\left(C_{m i c}\right)$ e relação porcentual entre essas duas variáveis, em razão dos sistemas de preparo do solo e das profundidades no ciclo da cultura da soja ${ }^{(1)}$.

\begin{tabular}{ccccc}
\hline $\begin{array}{c}\text { Profundidades } \\
(\mathrm{cm})\end{array}$ & Cerrado $^{(2)}$ & GR & SG & SD \\
\hline \multicolumn{5}{c}{ Carbono orgânico $\left(\mathrm{g} \mathrm{kg}^{-1} \mathrm{de} \mathrm{solo}\right)$} \\
$0-5$ & $17,0 \mathrm{a}$ & $14,9 \mathrm{~b}$ & $14,6 \mathrm{~b}$ & $15,4 \mathrm{~b}$ \\
$5-10$ & $14,8 \mathrm{a}$ & $13,7 \mathrm{a}$ & $13,7 \mathrm{a}$ & $14,0 \mathrm{a}$ \\
$10-20$ & $12,6 \mathrm{a}$ & $12,0 \mathrm{a}$ & $12,0 \mathrm{a}$ & $12,1 \mathrm{a}$ \\
$20-30$ & $10,8 \mathrm{a}$ & $9,8 \mathrm{~b}$ & $10,2 \mathrm{~b}$ & $10,0 \mathrm{~b}$ \\
$30-40$ & $8,5 \mathrm{a}$ & $8,2 \mathrm{~b}$ & $8,1 \mathrm{~b}$ & $8,2 \mathrm{~b}$ \\
\hline \multicolumn{5}{c}{ Carbono da biomassa microbiana $\left(\mathrm{mg} \mathrm{kg}^{-1} \mathrm{de} \mathrm{solo)}\right.$} \\
$0-5$ & $384,3 \mathrm{a}$ & $145,4 \mathrm{c}$ & $255,7 \mathrm{~b}$ & $222,1 \mathrm{bc}$ \\
$5-10$ & $339,9 \mathrm{a}$ & $134,8 \mathrm{~b}$ & $295,3 \mathrm{a}$ & $179,6 \mathrm{~b}$ \\
$10-20$ & $185,6 \mathrm{a}$ & $105,8 \mathrm{~b}$ & $229,2 \mathrm{a}$ & $103,2 \mathrm{~b}$ \\
$20-30$ & $45,6 \mathrm{c}$ & $75,1 \mathrm{~b}$ & $204,0 \mathrm{a}$ & $79,6 \mathrm{~b}$ \\
$30-40$ & $36,9 \mathrm{~b}$ & $47,6 \mathrm{~b}$ & $204,5 \mathrm{a}$ & $53,5 \mathrm{~b}$ \\
\hline \multicolumn{5}{c}{$\left(\mathrm{C}_{\text {mic }} \mathrm{C}_{\text {org }}\right)$} \\
$0-5$ & $2,3 \mathrm{a}$ & $1,0 \mathrm{c}$ & $1,8 \mathrm{ab}$ & $1,4 \mathrm{bc}$ \\
$5-10$ & $2,3 \mathrm{a}$ & $1,0 \mathrm{~b}$ & $2,1 \mathrm{a}$ & $1,3 \mathrm{~b}$ \\
$10-20$ & $1,5 \mathrm{a}$ & $0,9 \mathrm{~b}$ & $1,9 \mathrm{a}$ & $0,9 \mathrm{~b}$ \\
$20-30$ & $0,4 \mathrm{c}$ & $0,7 \mathrm{bc}$ & $1,9 \mathrm{a}$ & $0,8 \mathrm{~b}$ \\
$30-40$ & $0,4 \mathrm{~b}$ & $0,6 \mathrm{~b}$ & $2,5 \mathrm{a}$ & $0,7 \mathrm{~b}$ \\
\hline
\end{tabular}

(1)Para cada variável, médias seguidas pela mesma letra, na linha, não diferem entre si a 5\% de probabilidade pelo teste de Tukey; GR: uma a gradagens, SD: semeadura dire Cerrado sensu strictu. tidos por Pfenning et al. (1992) em Latossolo Vermelho-Amarelo na Amazônia, quando os valores do $\mathrm{C}_{\mathrm{mic}}$ nas camadas superficiais foram $100 \%$ maiores do que na camada $10-20 \mathrm{~cm}$.

Em todas as camadas do perfil, ocorreram diferenças estatísticas entre os tratamentos avaliados em relação ao $\mathrm{C}_{\text {mic }}$ (Tabela 3). Na camada de $0-5 \mathrm{~cm}$, o cerrado diferiu de todos os manejos. A GR foi semelhante ao SD e menor que o SG. Na camada de $5-10 \mathrm{~cm}$, e 10-20 cm, o SG foi semelhante ao cerrado; na camada de $20-30 \mathrm{~cm}$, a subsolagem apresentou os maiores valores $\left(204 \mathrm{mg} \mathrm{kg}^{-1}\right)$ e o cerrado os menores valores $\left(45,6 \mathrm{mg} \mathrm{kg}^{-1}\right)$. Na profundidade de $30-40 \mathrm{~cm}$, o SG diferiu dos outros tratamentos.

Antes do plantio da cultura, o $\mathrm{C}_{\text {mic }}: \mathrm{C}_{\text {org }}$ foi maior no cerrado e não houve diferenças significativas entre os preparos de solo (Tabela 1). Aos $30 \mathrm{DAG}$, houve diferenças significativas nos valores da razão $\mathrm{C}_{\text {mic }}: \mathrm{C}_{\mathrm{org}}$, em relação aos tipos de preparo do solo. Nas demais épocas, o cerrado apresentou diferença significativa em relação ao solo sob cultivo. Nesta época de amostragem, o preparo de solo $S G$ apresentou valor 6,9 vezes maior que o cerrado. Nas demais épocas, o cerrado apresentou diferença significativa em relação ao solo sob cultivo. Apenas na última amostragem, após a colheita da soja, a SD mostrou valor semelhante ao do cerrado. De acordo com Wardle (1994), a razão $\mathrm{C}_{\text {mic }}$ : $\mathrm{C}_{\text {org }}$ é um indicador da qualidade da matéria orgânica do solo.

Antes do plantio, a $\mathrm{C}_{\text {mic }}$ : $\mathrm{C}_{\text {org }}$ foi maior na profundidade de 5-10 cm e semelhante nas profundidades de 0-5 e 10-20 cm (Tabela 2). Aos 30 DAG, não houve diferenças significativas da $\mathrm{C}_{\text {mic }}: \mathrm{C}_{\text {org }}$ no perfil do solo. $\mathrm{Na}$ floração da cultura, a $\mathrm{C}_{\text {mic }}: \mathrm{C}_{\text {org }}$ foi maior nas profundidades 0-5, 5-10 e 10-20 cm. Após a colheita, os

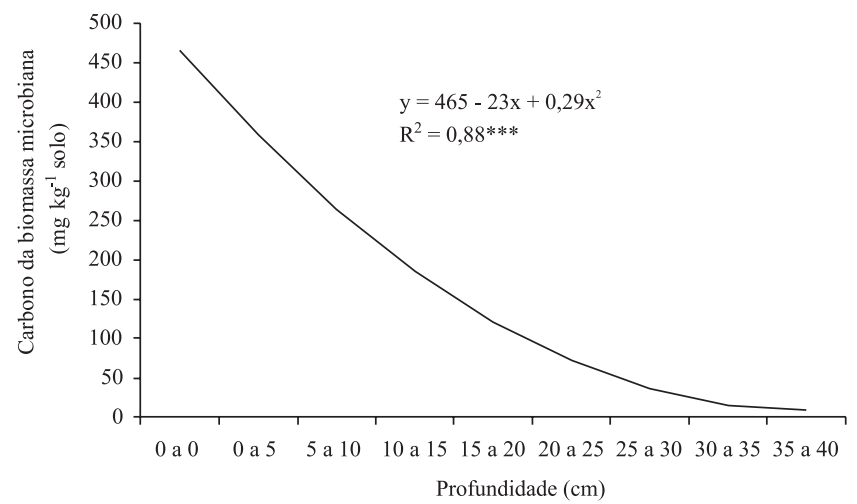

Figura 2. Relação entre o carbono da biomassa microbiana e a profundidade do solo no cerrado nativo. 
maiores valores foram encontrados nas profundidades de $0-5$ e $5-10 \mathrm{~cm}$.

Nas profundidades de $0-5,5-10$ e $10-20 \mathrm{~cm}$, a razão $\mathrm{C}_{\text {mic }}$ : $\mathrm{C}_{\text {org }}$ foi semelhante no cerrado e no SG (Tabela 3). Nas outras profundidades, no cerrado foi menor que na SG. $A$ razão $\mathrm{C}_{\text {mic }}$ : $\mathrm{C}_{\text {org }}$ na $\mathrm{GR}$ foi semelhante à da $\mathrm{SD}$, em todas as profundidades.

Houve correlação linear positiva e altamente significativa entre o $\mathrm{C}$ da biomassa microbiana e o C orgânico nos solos estudados, exceto na subsolagem (Tabela 4). $\mathrm{Na} S \mathrm{~S}$, o $\mathrm{C}_{\text {mic }}$ mostrou-se sensível na detecção das alterações dos processos do solo provocadas pelo revolvimento intensivo. Neste manejo, não houve correlação entre a $\mathrm{C}_{\text {mic }}: \mathrm{C}_{\text {org }}$ e os nutrientes estudados. Estas alterações refletem a eficiência da conversão do carbono microbiano, pela entrada dos resíduos orgânicos nos solos; as perdas do carbono do solo e a estabilização do carbono orgânico. Neste manejo não houve influência dos nutrientes na biomassa microbiana, mas houve alta correlação $(0,96 * * *)$ entre a $\mathrm{C}_{\text {mic }}: \mathrm{C}_{\text {org }}$ e o $\mathrm{C}_{\text {mic. }}$. Cattelan \& Vidor (1990) observaram correlações significativas da biomassa com os teores de P e K, com coeficientes de correlação de 0,686 e 0,675 , respectivamente. Segundo estes autores, a calagem e a adubação mineral ou orgânica favorecem o desenvolvimento microbiano de forma direta, pelo aumento do $\mathrm{pH}$ e pela disponibilidade de nutrientes às células dos microrganismos. A maior produção vegetal acarreta um aumento da atividade rizosférica e os resíduos orgânicos adicionados ao solo influenciam de forma indireta o crescimento microbiano.

No cerrado, houve correlação negativa do $\mathrm{pH}$ e positiva do Al com os atributos microbiológicos. Já nos sistemas de cultivo, estas correlações foram opostas. Isto sugere que, na área sob cerrado, possivelmente há grupos de microrganismos adaptados àquelas condições e com o preparo do solo, outros grupos de microrganismos podem ter se destacado, sugerindo que, para cada situação, grupos diferentes de microrganismos podem estar se adaptando às condições do solo. Em geral, a correlação entre os atributos químicos e microbiológicos foi semelhante nos sistemas GR e SD. Em determinadas condições, os atributos biológicos mostram o desenvolvimento de maturidade de ecossistemas influenciados pelo $\mathrm{pH}$, sugerindo especificidade nas atividades microbiológicas. $\mathrm{O}$ baixo $\mathrm{pH}$ pode ser um indicador de estresse ambiental refletindo na razão biomassa microbiana e carbono orgânico (Anderson \& Domsch, 1993). Em solos com pH mais baixos, compostos tóxicos são produzidos, ao contrário dos solos com $\mathrm{pH}$ mais alto (Vance et al., 1987). Em geral, houve correlação

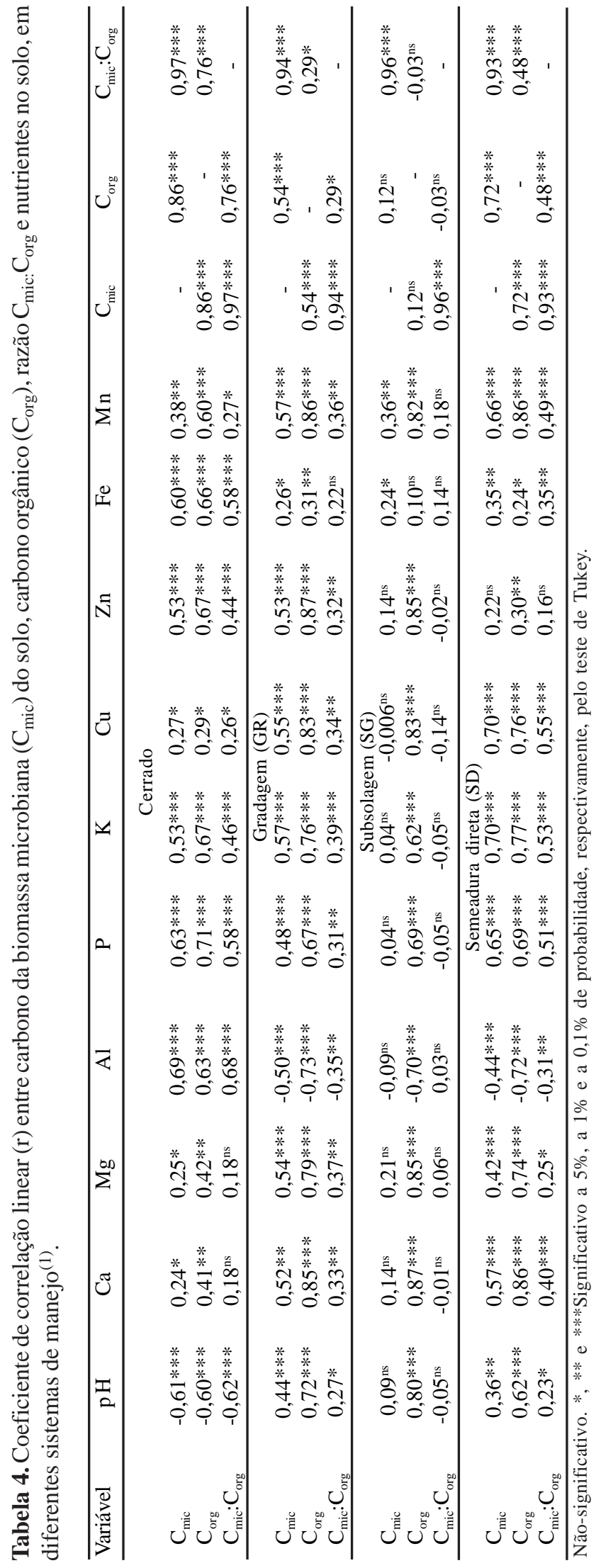


positiva entre o $\mathrm{C}_{\mathrm{mic}}$ e o $\mathrm{C}_{\mathrm{org}}$ com os micronutrientes, na maioria dos sistemas de manejo.

McGill et al. (1986) observaram que a disponibilidade de carbono é temporariamente maior em solos manejados organicamente. Os compostos orgânicos incrementam a biomassa microbiana, em comparação aos fertilizantes inorgânicos, porque ocorre aumento nas proporções de carbono e nitrogênio lábeis, estimulando diretamente a atividade da biomassa. Neste contexto, pode-se estabelecer maior diversidade de microrganis mos decompositores com maior eficiência na utilização do substrato, resultando assim numa maior relação $\mathrm{C}_{\text {mic }}: \mathrm{C}_{\text {org }}$ (Insam, 1990).

\section{Conclusões}

1. A subsolagem promove diminuição do carbono orgânico do solo após a colheita da soja.

2. As diferentes práticas de manejo alteram o carbono da biomassa do solo.

3. Na subsolagem não há correlação entre a relação carbono da biomassa microbiana:carbono orgânico e os nutrientes

4. Na semeadura direta, os valores de carbono da biomassa microbiana do solo são mais estáveis, principalmente na camada de $0-20 \mathrm{~cm}$.

\section{Referências}

ANDERSON, J.P.; DOMSCH, K.H. The metabolic quotient for $\mathrm{CO}_{2}\left(q \mathrm{CO}_{2}\right)$ as a specific activity parameter to asses the effects of environmental conditions, such as $\mathrm{pH}$, on the microbial biomass of forest soils. Soil Biology and Biochemistry, v.25, p.393-395, 1993. BAYER, C.; MIELNICZUK, J. Dinâmica e função da matéria orgânica. In: SANTOS, G.A ; CAMARGO, F.A.O. (Ed.). Fundamentos da matéria orgânica do solo: ecossistemas tropicai e subtropicais. Porto Alegre: Gênesis, 1999. p.9-26.

CATTELAN, A.J.; VIDOR, C. Flutuações na biomassa, atividade população microbiana do solo, em função de variações ambientais. Revista Brasileira de Ciência do Solo, v.14, p.133-142, 1990.

D'ANDRÉA, A.F.; SILVA, M.L.N.; CURI, N.; SIQUEIRA, M.A.C. Atributos biológicos indicadores da qualidade do solo em sistemas de manejo na região do Cerrado no sul do Estado de Goiás. Revista Brasileira de Ciência do Solo, v.26, p.913-923, 2002.

DE LUCA, T.H. Relationship of $0,5 \mathrm{M} \mathrm{K}_{2} \mathrm{SO}_{4}$ extractable anthronereactive carbon to indices of microbial activity in forest soils. Soil Biology and Biochemistry, v.30, p.1293-1299, 1998.

DORAN, J.W. Soil microbial and biochemical changes associated with reduced tillage. Soil Science Society of America Journal, v.44, p.765-771, 1980.
FRANZLUEBBERS, A.J.; HANEY, R.L.; HONS, F.M. Relationships of chloroform fumigation-incubation to soil organic matter pools. Soil Biology and Biochemistry, v.31, p.395-405, 1999.

GAMA-RODRIGUES, E.F. Biomassa microbiana e ciclagem de nutrientes. In: SANTOS, G.A.; CAMARGO, F.A.O. (Ed.). Fundamentos da matéria orgânica do solo: ecossistemas tropicais e subtropicais. Porto Alegre: Gênesis, 1999. p.227-243.

HINKELMAN, K.; KEMPTHORNE, O. Design and analysis of experiments: introduction to experimental design. New York: Wiley, 1994. v.1.

INSAM, H. Are the soil microbial biomass and basal respiration governed by the climatic regime? Soil Biology and Biochemistry, v.22, p.525-532, 1990.

JENKINSON, D.S.; POWLSON, D.S. The effects of biocidal treatments on metabolism in soil. Soil Biology Biochemistry, v.8, p.209-213, 1976

KAISER, E.A.; MARTENS, R.; HEINEMEYER, O. Temporal changes in soil microbial biomass carbon in an arable soil. Plant and Soil, v.170, p.287-295, 1995.

McGILL, W.B.; CANNON, K.R.; ROBERTSON, J.A.; COOK, F.D. Dynamics of soil microbial biomass and water-soluble organic $\mathrm{C}$ in Breton L after 50 years of cropping to two rotations. Canadian C in Breton L after 50 years of cropping to two

MILLIKEN, G.A.E.; JOHNSON, D.E. Analysis of messy data: designed experiments. New York: Chapman \& Hall, 1993. v.1.

MOREIRA, F.M.S.; SIQUEIRA, J.O. Microbiologia e bioquímica do solo. Lavras: Ufla, 2002. 625p.

PFENNING, L.; EDUARDO, B. de P.; CERRI, C.C. Os métodos da fumigação-incubação e fumigação-extração na estimativa da biomassa microbiana de solos da Amazônia. Revista Brasileira de Ciência do Solo, v.16, p.31-37, 1992

SAS INSTITUE (Cary, Estados Unidos). SAS statistical analysis system: user's guide, version 8. Cary, 1997. 295p.

SPARLING, G.P. Ratio of microbial biomass carbon to soil organic carbon as sensitive indicator of changes in soil organic matter. Australian Journal of Soil Research, v.30, p.195-207, 1992.

TISDALL, J.M.; OADES, J.M. Organic matter and water-stable aggregates in soils. Journal of Soil Science, v.33, p.141-163, 1982. URQUIAGA, S.; BODDEY, R.M.; NEVES, M.C.P. A necessidade de uma revolução mais verde. In: SIQUEIRA, J.O.; MOREIRA, F.M.S.; LOPES, A.S.; GUILHERME, L.R.G.; FAQUIN, V.; FURTINI NETO, A.E.; CARVALHO, J.G. (Ed.). Inter-relação fertilidade, biologia do solo e nutrição de plantas. Lavras: Ufla; Sociedade Brasileira de Ciência do Solo, 1999, p.175-181.

VANCE, E.D.; BROOKES, P.C.; JENKINSON, D.S. Microbial biomass measurements in forest soils: determination of $K c$ values bionass of hypotheses to explain the failure of the $K e$ values and test of hypotheses to explain the failure of the chloroform fumigation-incubation method in acid soils. Soil Biology and
Biochemistry, v.19, p.689-696, 1987. WALKLEY, A.; BLACK I.A. An examination of the degtiareff method for determining soil organic matter and a proposed modification of the chromic acid tritation method. Soil Science, v.37, p.29-38, 1934

WARDLE, D.A. Metodologia para quantificação da biomassa microbiana do solo. In: HUNGRIA, M.; ARAÚJO, R.S. (Ed.). Manual de métodos empregados em estudos de microbiologia agrícola. Brasília: Embrapa-CNPAF; Embrapa-CNPSo, 1994. p.419-436.

Recebido em 27 de outubro de 2003 e aprovado em 4 de março de 2004 